\title{
BROWDER'S TYPE STRONG CONVERGENCE THEOREM FOR $S$-NONEXPANSIVE MAPPINGS
}

\author{
Jong Kyu Kim, Daya Ram Sahu, and Sajid Anwar
}

\begin{abstract}
We prove a common fixed point theorem for $S$-contraction mappings without continuity. Using this result we obtain an approximating curve for $S$-nonexpansive mappings in a Banach space and prove Browder's type strong convergence theorem for this approximating curve. The demiclosedness principle for $S$-nonexpansive mappings is also established.
\end{abstract}

\section{Introduction}

Let $C$ be a subset of a normed space $X$. A mapping $T: C \rightarrow X$ is said to be nonexpansive if

$$
\|T x-T y\| \leq\|x-y\|
$$

for all $x, y \in C$. Suppose $S: C \rightarrow X$ is a mapping. Then $T$ is said to be $S$-nonexpansive if

$$
\|T x-T y\| \leq\|S x-S y\|
$$

for all $x, y \in C$. The class of $S$-nonexpansive mappings is a generalization of that of nonexpansive mappings [7].

In 1967, Browder [2] proved the following strong convergence theorem for nonexpansive mappings: Let $C$ be a nonempty closed convex bounded subset of a Hilbert space $H$ and $T: C \rightarrow C$ a nonexpansive self-mapping. Let $u \in C$ and for each $t \in(0,1)$, let

$$
G_{t} x=t u+(1-t) T x, x \in C .
$$

Then $G_{t}$ has a unique fixed point $x_{t}$ in $C$ and $x_{t}$ converges strongly to a fixed point of $T$ in $C$ as $t \rightarrow 0$.

On the other hand, Shahzad [8] introduced the notion of $R$-subweakly commutativity which provides existence of a curve $\left\{x_{\lambda}\right\}$ in $C$ defined by

$$
x_{\lambda}=S x_{\lambda}=(1-\lambda) q+\lambda T x_{\lambda} \text { for all } \lambda \in(0,1)
$$

Received October 16, 2008.

2000 Mathematics Subject Classification. 47H09, 46B20, 47H10, 54H25.

$K e y$ words and phrases. demicontinuity, $R$-weakly commutativity, $S$-contraction mapping, $S$-nonexpansive mapping.

The first author was supported by the Kyungnam University Research Fund 2008.

(C)2010 The Korean Mathematical Society 
for $S$-nonexpansive mappings (see Proposition 3.3).

In this paper, an important existence result as a significant improvement of a result of Shahzad [8] for $S$-contraction mappings without continuity is proved. This result is applied for existence of approximating curve $\left\{x_{\lambda}\right\}$ defined by (1.1) and existence of fixed points of $S$-nonexpansive mappings without continuity. Also we prove demiclosedness principle for $S$-nonexpansive mappings and prove strong convergence of curve $\left\{x_{\lambda}\right\}$ in a reflexive Banach space with a weakly continuous duality mapping. Our results are significant improvements of corresponding results of Al-Thagafi [1], Dotson [3], Jungck [4] and Shahzad [8]. One of our results is an extension of celebrated result of Browder [2] from Hilbert space to Banach space for the class of $S$-nonexpansive mappings.

\section{Preliminaries}

Let $(X, d)$ be a metric space, $C$ a nonempty subset of $X$ and let $S, T: C \rightarrow C$ be two mappings. Throughout this paper, we adopt the following notations:

$$
\mathcal{C}_{S, T}=\{u \in C: S u=T u\}
$$

and

$$
F(T)=\{u \in C: T u=u\} .
$$

The pair $\{S, T\}$ is said to be $R$-weakly commuting on $C$ [6] if there exists $R>0$ such that

for all $x \in C$.

$$
d(S T x, T S x) \leq R d(S x, T x)
$$

Let $C$ be a nonempty subset of a normed space $X$. The set $C$ is called $q$-starshaped with $q \in C$ if for all $x \in C$, the segment $[x, p]$ joining $x$ to $q$ is contained in $C$, that is, $t x+(1-t) q \in C$ for all $x \in C$ and $0 \leq t \leq 1$. Note that if $\mathrm{S}$ is $q$-starshaped for every $q \in C$, then $C$ is convex.

Let $C$ be a nonempty $q$-starshaped subset of a normed space $X$. Then a mapping $S: C \rightarrow C$ is said to be $q$-affine if

$$
t S x+(1-t) S q \in C
$$

for all $x \in C$ and $0 \leq t \leq 1$.

Definition 2.1. Let $C$ be a nonempty subset of a normed space $X$ and let $S, T: C \rightarrow C$ be two mappings such that $F(S) \neq \emptyset$. Suppose $q \in F(S)$ and $C$ is $q$-starshaped. Then $S$ and $T$ are called $R$-subweakly commuting on $C$ if there exists a real number $R>0$ such that

$$
\|S T x-T S x\| \leq R d(S x,[T x, q])
$$

for all $x \in C$, where $d(y, D)=\inf \{\|y-z\|: z \in D\}$ for $D \subseteq C$ and $y \in C$.

It is clear from Definition 2.1 that commutativity implies $R$-subweak commutativity, but the converse is not true in general (see, example in [8]).

Let $C$ be a nonempty subset of a normed space $X$ and $T: C \rightarrow C$ a mapping. When $\left\{x_{n}\right\}$ is a sequence in $X$, we denote the strong convergence of $\left\{x_{n}\right\}$ to 
$x$ by $x_{n} \rightarrow x$, the weak convergence of $\left\{x_{n}\right\}$ to $x$ by $x_{n} \rightarrow x$ and the weak* convergence of $\left\{x_{n}\right\}$ to $x$ by $x_{n} \rightarrow^{*} x$. T is said to be demicontinuous if $\left\{x_{n}\right\}$ is a sequence in $X$ such that $x_{n} \rightarrow x$, then $T x_{n} \rightarrow T x$. The mapping $T$ is said to be weakly continuous if $\left\{x_{n}\right\}$ is a sequence in $X$ such that $x_{n} \rightarrow x$, then $T x_{n} \rightarrow T x$. Note that every continuous mapping is demicontinuous.

Let $X$ be a Banach space. A mapping $T$ with domain $D(T)$ and range $R(T)$ in $X$ is said to be demiclosed at a point $y \in R(T)$ if whenever $\left\{x_{n}\right\}$ is a sequence in $D(T)$ which converges weakly to a point $u \in D(T)$ and $\left\{T x_{n}\right\}$ converges strongly to $y$, then $T u=y$.

A Banach space $X$ is said to satisfy the Opial condition ([5]) if for each sequence $\left\{x_{n}\right\}$ in $X$ which converges weakly to a point $x \in X$, we have

$$
\liminf _{n \rightarrow \infty}\left\|x_{n}-x\right\|<\liminf _{n \rightarrow \infty}\left\|x_{n}-y\right\|
$$

for all $y \in X, y \neq x$.

Let $X$ be a Banach space. Then a mapping $J: X \rightarrow 2^{X^{*}}$ defined by

$$
J(u)=\left\{j \in X^{*}:\langle u, j\rangle=\|u\|^{2},\|j\|=\|u\|\right\}
$$

is called the normalized duality mapping. Suppose that $J$ is single-valued. Then $J$ is said to be weakly sequentially continuous if, for each $\left\{x_{n}\right\}$ in $X$ with $x_{n} \rightarrow x, J\left(x_{n}\right) \rightarrow^{*} J(x)$. It is well known that if $X$ admits a weakly sequentially continuous duality mapping, then $X$ satisfies the Opial condition.

\section{Auxiliary results}

The following lemma is an improvement of Lemma 2.1 of Shahzad [8] in the following ways:

(i) $C$ is not necessarily closed,

(ii) $T$ is not necessarily continuous,

(iii) location of unique common fixed point is given.

Lemma 3.1. Let $(X, d)$ be a metric space and $C$ a nonempty subset of $X$. Let $S, T: C \rightarrow C$ be two mappings such that

(i) $T(C) \subseteq S(C)$,

(ii) $T$ is $S$-contraction, i.e., there exists a constant $k \in(0,1)$ such that

$$
\|T x-T y\| \leq k\|S x-S y\| \text { for all } x, y \in C,
$$

(iii) $\{S, T\}$ is $R$-weakly commuting on $C$.

Then we have the following:

(a) $F(S) \cap F(T) \cap T(C)$ is a singleton if $T(C)$ is complete,

(b) $F(S) \cap F(T) \cap S(C)$ is a singleton if $S(C)$ is complete.

Proof. Pick $x_{0} \in C$. Since $T(C) \subseteq S(C)$, we can construct a sequence $\left\{x_{n}\right\}$ in $C$ such that $S x_{n}=T x_{n-1}$ for all $n \in \mathbb{N}$. Then

$$
d\left(S x_{n+1}, S x_{n}\right)=d\left(T x_{n}, T x_{n-1}\right) \leq k d\left(S x_{n}, S x_{n-1}\right) \text { for all } n \in \mathbb{N},
$$

it follows that $\left\{S x_{n}\right\}$ is a Cauchy sequence in $C$. 
(a) If $T(C)$ is complete, there exists a point $z \in T(C)$ such that $T x_{n} \rightarrow z \in$ $T(C)$. Thus, $S x_{n} \rightarrow z$. Since $z \in T(C) \subseteq S(C)$, there exists $u \in C$ such that $z=S u$. By the $S$-contractivity of $T$, we have

$$
d\left(T u, T x_{n}\right) \leq k d\left(S u, S x_{n}\right) .
$$

Taking limit as $n \rightarrow \infty$ yields

$$
d(T u, z) \leq k d(z, z)=0 .
$$

Thus, $S u=T u=z$. Since $\{S, T\}$ is $R$-weakly commuting on $C$, it follows that $S z=T z$. Note that

$$
d\left(T z, T x_{n}\right) \leq k d\left(S z, S x_{n}\right) .
$$

Letting limit as $n \rightarrow \infty$, we obtain

$$
d(T z, z) \leq k d(S z, z)=k d(T z, z) .
$$

It shows that $S z=T z=z$. By the $S$-contractivity of $T$, we conclude that $F(S) \cap F(T) \cap T(C)=\{z\}$.

(b) Suppose $S(C)$ is complete. Then $S x_{n} \rightarrow z$ for some $z \in S(C)$ and there exist $u \in C$ such that $z=S u$. As part (a) we can show that $S z=T z=z$. The $S$-contractivity of $T$ implies that $F(S) \cap F(T) \cap S(C)=\{z\}$.

Before presenting existence results, we establish the demiclosedness principle for $S$-nonexpansive nonself mappings.

Proposition 3.2 (Demiclosedness principle). Let $X$ be a Banach space satisfying the Opial condition and $C$ a nonempty weakly closed subset of $X$. Let $S, T: C \rightarrow X$ be two mappings such that $S$ is weakly continuous and $T$ is $S$-nonexpansive. Then $S-T$ is demiclosed on $C$, i.e., if $\left\{x_{n}\right\}$ is a sequence in $C$ such that $x_{n} \rightarrow u \in C$ and $(S-T) x_{n} \rightarrow y$, then $(S-T) u=y$.

Proof. Let $\left\{x_{n}\right\}$ be a sequence in $C$ such that $x_{n} \rightarrow u \in C$ and $\lim _{n \rightarrow \infty} \|(S-$ $T) x_{n}-y \|=0$ for some $y \in X$. We show that $(S-T) u=y$. Suppose, for contradiction, that $(S-T) u \neq y$. Observe that

$$
\left\|S x_{n}-T u-y\right\| \leq\left\|S x_{n}-T x_{n}-y\right\|+\left\|T x_{n}-T u\right\|,
$$

which implies that

$$
\liminf _{n \rightarrow \infty}\left\|S x_{n}-T u-y\right\| \leq \liminf _{n \rightarrow \infty}\left\|S x_{n}-S u\right\| .
$$

By the weak continuity of $S$, we obtain that $S x_{n} \rightarrow S u \in C$. By the Opial condition, we have

$$
\begin{aligned}
\liminf _{n \rightarrow \infty}\left\|S x_{n}-S u\right\| & <\liminf _{n \rightarrow \infty}\left\|S x_{n}-T u-y\right\| \\
& \leq \liminf _{n \rightarrow \infty}\left\|S x_{n}-S u\right\|,
\end{aligned}
$$

a contradiction. Therefore, $(S-T) u=y$. 
Proposition 3.3. Let $C$ be a nonempty subset of a normed space $X$. Let $S, T: C \rightarrow C$ be two mappings such that

(i) $T$ is $S$-nonexpansive and $S$ is q-affine with $q \in F(S)$,

(ii) $T(C) \subseteq S(C)$ and $C$ is q-starshaped,

(iii) $\{S, T\}$ is $R$-subweakly commuting,

(iv) $S(C)$ is complete.

Then there exists exactly one point $x_{\lambda} \in S(C)$ such that

$$
x_{\lambda}=S x_{\lambda}=(1-\lambda) q+\lambda T x_{\lambda}
$$

for all $\lambda \in(0,1)$.

Proof. For each $\lambda \in(0,1)$, define a mapping $T_{\lambda}$ by

$$
T_{\lambda} x=(1-\lambda) q+\lambda T x
$$

for all $x \in C$. Note that each $T_{\lambda}: C \rightarrow C$ is an $S$-contraction on $C$. Indeed,

$$
\begin{aligned}
\left\|T_{\lambda} x-T_{\lambda} y\right\| & =\lambda\|T x-T y\| \\
& \leq \lambda\|S x-S y\|
\end{aligned}
$$

for all $x, y \in C$. Since $\{S, T\}$ is $R$-subweakly commuting and $S$ is $q$-affine, we have

$$
\begin{aligned}
\left\|S T_{\lambda} x-T_{\lambda} S x\right\| & =\|[(1-\lambda) q+\lambda S T x]-[(1-\lambda) q+\lambda T S x]\| \\
& =\lambda\|T S x-S T x\| \\
& \leq \lambda R\left\|S x-T_{\lambda} x\right\|
\end{aligned}
$$

for all $x \in C$. Thus, the pair $\left\{S, T_{\lambda}\right\}$ is $R$-weakly commuting on $C$.

For $x \in C$, we have $T x \in T(C) \subseteq S(C)$, i.e., there exists a point $y \in C$ such that $T x=S y \in S(C)$. Observe that

$$
T_{\lambda} x=(1-\lambda) q+\lambda T x=(1-\lambda) q+\lambda S y \in S(C) .
$$

It follows that $T_{\lambda}(C) \subseteq S(C)$ for all $\lambda \in(0,1)$.

For each $\lambda \in(0,1)$, we conclude that
(i) ${ }^{\prime} T_{\lambda}(C) \subseteq S(C)$,
(ii) ${ }^{\prime} T_{\lambda}$ is $S$-contraction,
(iii)' $S(C)$ is complete,
(iv) $)^{\prime}\left\{S, T_{\lambda}\right\}$ is $R$-weakly commuting on $C$.

Therefore, Lemma 3.1 implies that there exists exactly one point $x_{\lambda} \in S(C)$ such that $x_{\lambda}=S x_{\lambda}=T_{\lambda} x_{\lambda}$.

The main results of this section are the following:

Theorem 3.4. Let $C$ be a nonempty subset of a normed space $X$. Let $S, T$ : $C \rightarrow C$ be two mappings satisfy the conditions (i) $\sim$ (iii) of Proposition 3.3. Suppose $S(C)$ is compact. Then we have the following:

(a) There exists $y \in S(C)$ such that $S y=T y$.

(b) If $S$ or $T$ is demicontinuous, then $y \in F(S) \cap F(T)$. 
Proof. Let $\left\{\lambda_{n}\right\}$ be a sequence in $(0,1)$ such that $\lambda_{n} \rightarrow 1$. By Proposition 3.3, there exists exactly one point $x_{\lambda_{n}} \in S(C)$ such that

$$
x_{\lambda_{n}}=S x_{\lambda_{n}}=\left(1-\lambda_{n}\right) q+\lambda_{n} T x_{\lambda_{n}} \text { for all } n \in \mathbb{N} .
$$

Set $x_{\lambda_{n}}:=x_{n}$. By the compactness of $S(C)$, there exists a subsequence $\left\{\lambda_{m}\right\}$ of $\left\{\lambda_{n}\right\}$ such that $\lim _{m \rightarrow \infty} S x_{m}=y \in S(C)$. Thus, $y=S u$ for some $u \in C$. The assumption (ii) implies that $\left\{T x_{m}\right\}$ is bounded. It follows that

$$
\left\|x_{m}-T x_{m}\right\| \leq\left(1-\lambda_{m}\right)\left\|q-T x_{m}\right\| \rightarrow 0 \text { as } m \rightarrow \infty .
$$

This gives that $\lim _{m \rightarrow \infty} T x_{m}=y$. By $S$-nonexpansiveness of $T$, we have

$$
\left\|T x_{m}-T u\right\| \leq\left\|S x_{m}-S u\right\|=\left\|S x_{m}-y\right\| .
$$

Taking limit as $m \rightarrow \infty$ yields $T u=y$.

(a) Since $\{S, T\}$ is $R$-weakly commuting, it follows that $S y=T y$.

(b) Suppose $S$ is demicontinuous. Since $\lim _{m \rightarrow \infty} x_{m}=\lim _{m \rightarrow \infty} S x_{m}=y$, it follows from the demicontinuity of $S$ that $S y=y$. Thus, we conclude from $S y=T y$ that $y \in F(S) \cap F(T)$. Similarly, we can prove that $y \in F(S) \cap F(T)$ when $T$ is demicontinuous.

Example 3.5. Let $X=[0,1]$ with the usual metric and $C=X$. Define

$$
S x=\left\{\begin{array}{ll}
0 & \text { if } \quad x \in[0,1), \\
1 & \text { if } \quad x=1
\end{array} \text { and } T x=0 \text { for all } x \in C .\right.
$$

Then all hypotheses of Theorem 3.4 are satisfied. Note that $0 \in F(S) \cap F(T)$.

Remark 3.6. The mapping $S$ in Theorem 3.4 is not necessarily linear. Therefore, Theorem 3.4 improves Theorem 2.2 of Al-Thagafi [1], Theorem 1 of Dotson [3], Theorem 3.1 of Jungck [4] and Lemma 2.2 of Shahzad [8].

Theorem 3.7. Let $C$ be a nonempty subset of a Banach space $X$. Let $S, T$ : $C \rightarrow C$ be two mappings satisfying conditions (i) $\sim$ (iii) of Proposition 3.3. Suppose $S(C)$ is weakly compact. Then $F(S) \cap F(T) \neq \emptyset$ if one of the following conditions holds:

$\left(C_{1}\right) I-T$ is demiclosed at zero.

$\left(C_{2}\right) X$ satisfies the Opial condition.

Proof. Let $\left\{\lambda_{n}\right\}$ be a sequence in $(0,1)$ with $\lambda_{n} \rightarrow 1$. Since $S(C)$ is weakly compact, it follows that $S(C)$ is norm-closed and hence it is complete. Then form Proposition 3.3, there exists exactly one point $x_{n}$ such that

$$
x_{n}=S x_{n}=\left(1-\lambda_{n}\right) q+\lambda_{n} T x_{n} \text { for all } n \in \mathbb{N} .
$$

The condition $T(C) \subseteq S(C)$ implies that $T(C)$ is bounded and hence $x_{n}-$ $T x_{n} \rightarrow 0$. By the weak compactness of $S(C)$, there exists a subsequence $\left\{x_{n_{i}}\right\}$ of $\left\{x_{n}\right\}$ such that $x_{n_{i}} \rightarrow v$. The weak continuity of $S$ implies that $v=S v$.

If $I-T$ is demiclosed at zero, then $v=T v$ and hence theorem is proved. If we assume that $X$ satisfies the Opial condition, then we can conclude form Proposition 3.2 that $S v=T v$. 
Remark 3.8. The main results of this section can be extended in complete p-normed spaces.

\section{Browder's type strong convergence theorem}

The following result extends Browder's strong convergence theorem for $S$ nonexpansive mappings.

Theorem 4.1. Let $X$ be a reflexive Banach space with a weakly continuous duality mapping $J: X \rightarrow X^{*}$. Let $C$ be a weakly closed subset of $X, S: C \rightarrow C$ a affine weakly continuous mapping with $q \in F(S)$ such that $C$ is q-starshaped. Let $T: C \rightarrow C$ be an $S$-nonexpansive mapping with $\mathcal{C}_{S, T} \neq \emptyset$ and let $\left\{x_{\lambda}: \lambda \in\right.$ $(0,1)\}$ be the approximating curve in $C$ defined by $(3.1)$. Then $\lim _{\lambda \rightarrow 1} x_{\lambda}=\tilde{x}$ exists and $\tilde{x} \in F(S) \cap F(T)$.

Proof. First, we show that $\left\{x_{\lambda}\right\}$ is bounded. Let $p \in \mathcal{C}_{S, T}$. Then $S p=T p=u$ for some $u \in C$. From (3.1), we have

$$
\begin{aligned}
\left\|x_{\lambda}-u\right\| & \leq(1-\lambda)\|q-u\|+\lambda\left\|T x_{\lambda}-T p\right\| \\
& \leq(1-\lambda)\|q-u\|+\lambda\left\|S x_{\lambda}-S p\right\| \\
& =(1-\lambda)\|q-u\|+\lambda\left\|x_{\lambda}-u\right\|,
\end{aligned}
$$

which implies that

$$
\left\|x_{\lambda}-u\right\| \leq\|q-u\|
$$

Thus, $\left\{x_{\lambda}\right\}$ is bounded. Assume that $\left\{\lambda_{n}\right\}$ is a sequence in $(0,1)$ such that $\lim _{n \rightarrow \infty} \lambda_{n}=1$ and $\left\{x_{\lambda_{n}}\right\}$ is bounded. Since $X$ is reflexive, we may assume that $x_{\lambda_{n}} \rightarrow v \in C$. Set $x_{\lambda_{n}}:=x_{n}$. Again, from (3.1), we have

$$
\left\|x_{n}-T x_{n}\right\| \leq\left(1-\lambda_{n}\right)\left\|q-T x_{n}\right\| \rightarrow 0 .
$$

On the other hand, we have

$$
\begin{aligned}
\left\langle x_{\lambda}-T x_{\lambda}, J\left(x_{\lambda}-u\right)\right\rangle & =\left\langle x_{\lambda}-u+T p-T x_{\lambda}, J\left(x_{\lambda}-u\right)\right\rangle \\
& =\left\|x_{\lambda}-u\right\|^{2}-\left\langle T x_{\lambda}-T p, J\left(x_{\lambda}-p\right)\right\rangle \\
& \geq\left\|x_{\lambda}-u\right\|^{2}-\left\|T x_{\lambda}-T p\right\|\left\|x_{\lambda}-u\right\| \\
& \geq\left\|x_{\lambda}-u\right\|^{2}-\left\|S x_{\lambda}-S p\right\|\left\|x_{\lambda}-u\right\| \\
& =0 .
\end{aligned}
$$

Since $x_{\lambda}-T x_{\lambda}=\frac{1-\lambda}{\lambda}\left(q-x_{\lambda}\right)$, it follows from (4.1) that

$$
\left\langle x_{\lambda}-q, J\left(x_{\lambda}-u\right)\right\rangle \leq 0 .
$$

Since $S$ is weakly continuous, $x_{n} \rightarrow v \in C$ and $S x_{n}-T x_{n} \rightarrow 0$, we obtain from Proposition 3.2 that $S v=T v$. Suppose that $S v=T v=w$ for some $w \in C$. Using (4.2), we get

$$
\left\langle x_{n}-q, J\left(x_{n}-w\right)\right\rangle \leq 0 .
$$


From (4.3), we have

$$
\begin{aligned}
\left\|x_{n}-w\right\|^{2} & =\left\langle x_{n}-w, J\left(x_{n}-w\right)\right\rangle \\
& =\left\langle x_{n}-q, J\left(x_{n}-w\right)\right\rangle+\left\langle q-w, J\left(x_{n}-w\right)\right\rangle \\
& \leq\left\langle q-w, J\left(x_{n}-w\right)\right\rangle .
\end{aligned}
$$

Since $J$ is weakly continuous, it follows from (4.4) that $x_{n} \rightarrow w$ as $n \rightarrow \infty$. Note that the weak continuity of $S$ implies that $x_{n}=S x_{n} \rightarrow S w$. By the uniqueness of weak limit of $\left\{x_{n}\right\}$, we have $S w=w$. Observe that

$$
\begin{aligned}
\|w-T w\| & \leq\left\|w-x_{n}\right\|+\left\|x_{n}-T x_{n}\right\|+\left\|T x_{n}-T w\right\| \\
& \leq\left\|w-x_{n}\right\|+\left\|x_{n}-T x_{n}\right\|+\left\|S x_{n}-S w\right\| \\
& \leq 2\left\|w-x_{n}\right\|+\left\|x_{n}-T x_{n}\right\| \rightarrow 0 \text { as } n \rightarrow \infty .
\end{aligned}
$$

Thus, $w=S w=T w$.

Now, it remains to prove that the approximating curve $\left\{x_{\lambda}\right\}$ converges strongly to $w$. Suppose, for contradiction, that there exists another sequence $\left\{x_{\lambda_{n^{\prime}}}\right\} \subset\left\{x_{\lambda}\right\}$ such that $x_{\lambda_{n^{\prime}}} \rightarrow w^{\prime} \neq w$ as $\lambda_{n^{\prime}} \rightarrow 1$. Then, we have $w^{\prime} \in F(S) \cap F(T)$. From (4.2), we have

$$
\left\langle x_{\lambda}-q, J\left(x_{\lambda}-p\right)\right\rangle \leq 0 \text { for all } p \in F(S) \cap F(T) .
$$

Using (4.5), we have

$$
\left\langle w-q, J\left(w-w^{\prime}\right)\right\rangle \leq 0 \text { and }\left\langle q-w^{\prime}, J\left(w-w^{\prime}\right)\right\rangle=\left\langle w^{\prime}-q, J\left(w^{\prime}-w\right)\right\rangle \leq 0 .
$$

Adding these two inequalities, we obtain

$$
\left\|w-w^{\prime}\right\|^{2}=\left\langle w-w^{\prime}, J\left(w-w^{\prime}\right)\right\rangle \leq 0 .
$$

Thus, $w=w^{\prime}$. Therefore, $\lim _{\lambda \rightarrow 1} x_{\lambda}$ exists and $\lim _{\lambda \rightarrow 1} x_{\lambda}=w \in F(S) \cap$ $F(T)$.

Remark 4.2. Nonemptyness of $\mathcal{C}_{S, T}$ can be replaced by boundedness of $T(C)$.

\section{References}

[1] M. A. Al-Thagafi, Common fixed points and best approximation, J. Approx. Theory 85 (1996), no. 3, 318-323.

[2] F. E. Browder, Convergence of approximants to fixed points of nonexpansive non-linear mappings in Banach spaces, Arch. Rational Mech. Anal. 24 (1967), 82-90.

[3] W. G. Dotson, Jr., Fixed point theorems for non-expansive mappings on star-shaped subsets of Banach spaces, J. London Math. Soc. (2) 4 (1972), 408-410.

[4] G. Jungck, Coincidence and fixed points for compatible and relatively nonexpansive maps, Internat. J. Math. Math. Sci. 16 (1993), no. 1, 95-100.

[5] Z. Opial, Weak convergence of the sequence of successive approximations for nonexpansive mappings, Bull. Amer. Math. Soc. 73 (1967), 591-597.

[6] R. P. Pant, Common fixed points of noncommuting mappings, J. Math. Anal. Appl. 188 (1994), no. 2, 436-440.

[7] S. Park, On f-nonexpansive maps, J. Korean Math. Soc. 16 (1979), no. 1, 29-38.

[8] N. Shahzad, Invariant approximations and R-subweakly commuting maps, J. Math. Anal. Appl. 257 (2001), no. 1, 39-45. 
Jong KYU KIM

Department of Mathematics Educations

KYUNGNAM University

KYUNGNAM 631-701, KoREA

E-mail address: jongkyuk@kyungnam.ac.kr

Daya Ram Sahu

Department of Mathematics

BANARAS HINDU UNIVERSITY

VARANASI-221005, INDIA

E-mail address: drsahu@bhu.ac.in

SAJID ANWAR

Anjuman College of Engineering and Technology

Mangalwari Bazar Road, SAdar, NAGPur-1, India

E-mail address: sajid_anwar@sify.com 